\title{
Ageing-Associated Oxidative Stress and Inflammation Are Alleviated by Products from Grapes
}

\author{
K. S. Petersen and C. Smith \\ Department of Physiological Sciences, Stellenbosch University, Stellenbosch 7600, South Africa \\ Correspondence should be addressed to C. Smith; csmith@sun.ac.za
}

Received 23 October 2015; Revised 3 February 2016; Accepted 7 February 2016

Academic Editor: Hesham A. El Enshasy

Copyright ( 92016 K. S. Petersen and C. Smith. This is an open access article distributed under the Creative Commons Attribution License, which permits unrestricted use, distribution, and reproduction in any medium, provided the original work is properly cited.

\begin{abstract}
Advanced age is associated with increased incidence of a variety of chronic disease states which share oxidative stress and inflammation as causative role players. Furthermore, data point to a role for both cumulative oxidative stress and low grade inflammation in the normal ageing process, independently of disease. Therefore, arguably the best route with which to address premature ageing, as well as age-associated diseases such as diabetes, cardiovascular disease, and dementia, is preventative medicine aimed at modulation of these two responses, which are intricately interlinked. In this review, we provide a detailed account of the literature on the communication of these systems in the context of ageing, but with inclusion of relevant data obtained in other models. In doing so, we attempted to more clearly elucidate or identify the most probable cellular or molecular targets for preventative intervention. In addition, given the absence of a clear pharmaceutical solution in this context, together with the everincreasing consumer bias for natural medicine, we provide an overview of the literature on grape (Vitis vinifera) derived products, for which beneficial effects are consistently reported in the context of both oxidative stress and inflammation.
\end{abstract}

\section{Introduction}

With ageing, the capacity of the body to function optimally declines. There is a combination of genetic and lifestyle factors which may either accelerate or slow down the ageing process. A number of chronic diseases are associated with advanced age: these include cardiovascular disease (CVD), diabetes, metabolic syndrome, and Alzheimer's disease. This results in an exponential increase in the disease burden on modern society, relative to a few decades ago, due to longer life expectancy. The World Health Organisation states that from 2015 to 2050, the proportion of the world's population over 60 years will increase from $12 \%$ to $22 \%$ [1]. Taking into consideration that predominant research in the field of age-associated diseases considers the sixth decade of life to be a risk factor for the rapid progression and onset of ageassociated diseases, the disease burden will almost double in the next 35 years. It is thus vital not only to elucidate the causes and progression of these chronic conditions, but also to actively search and investigate potential preventative therapies that may slow the processes contributing to physiological ageing.

Although the ageing-associated chronic disease states are each uniquely complex in terms of their aetiology, development, and progression, they do share common aetiologies which stem from two main entities, namely, cumulative oxidative stress and chronic inflammation. Briefly, oxidants are produced by normal cell metabolism and various physiological responses. However, when the production of oxidants outweighs the capacity of endogenous antioxidant systems, oxidative stress is incurred. Furthermore, while inflammation is crucial for repair of tissue injury and primary defence against invading pathogens and chemicals, it also results in unintended detriment to previously uninjured cells. Although these are necessary systems in the body, both oxidative stress and the inflammatory response, if unchecked, can have detrimental consequences which have been linked to accelerated ageing and the progression of 
age-associated disease. We postulated that the effects of the inflammatory immune system and oxidative stress on allostatic load are interlinked. This has led us to investigate the potential of antioxidants as treatment options to attenuate the cumulative effects of both oxidative stress and chronic low grade inflammation. Given the modern consumer bias for natural medicines, we focused on a group of plant medicines which are consistently associated with beneficial effects on these processes in the literature, grape-derived polyphenols. In this review, we will provide a more in-depth review of interconnected molecular mechanisms of oxidative stress and inflammation in the physiological ageing process, before moving our focus to a discussion of the merits of these plant medicines as potential preventative therapy in this context.

\section{Contribution of Oxidative Stress to Premature Ageing}

In the context of ageing, reactive oxygen and nitrogen species (RONS) are generally the major molecules which contribute significantly to oxidative stress. The most frequently studied free radicals are superoxide $\left(\mathrm{O}_{2}{ }^{-}\right)$, hydrogen peroxide $\left(\mathrm{H}_{2} \mathrm{O}_{2}\right)$, hydroxyl radical $\left({ }^{\circ} \mathrm{OH}\right)$, peroxynitrite $\left(\mathrm{ONO}_{2}{ }^{-}\right)$, and nitric oxide (NO). The generation of these free radicals is necessary as they are essential for host defence: phagocytic cells use ROS to digest invading pathogens and debris. Furthermore, they act as signalling molecules, regulating cell growth and apoptosis, adhesion, and differentiation. More specifically, RONS are formed during processes such as the mitochondrial electron transport chain as well as enzyme systems such as cytochrome P450, lipoxygenase and cyclooxygenase, the NADPH-oxidase complex, xanthine oxidase, and peroxisomes [2]. In contrast to these roles in growth, repair, and immune functions which are all beneficial to the host, these molecules also have the ability to oxidise signalling molecules, DNA, macromolecules, and cell structures such as lipid membranes of healthy host cells, all of which are to the detriment of these cells. Usually, each cell has defence mechanisms to counteract the occurrence of oxidative stress. These endogenous enzymatic antioxidant defences include superoxide dismutase (SOD), glutathione peroxidase, catalases, glutathione/TrxR, and peroxiredoxins. An appropriately nutritious diet is vital to maintain these systems, as these natural antioxidants are supplemented or replenished by antioxidant constituents of various fruits and vegetables. It is only when the capacity of the body's antioxidant defences is outweighed by the rate of production of free radicals that oxidative stress is incurred by unquenched free radicals which can alter surrounding cell structures and environment.

With advancing age, various factors, among these is a natural progressive decline in endogenous antioxidant capacity, cause disruption in the balance between pro- and antioxidant mechanisms and RONS accumulate beyond the normal endogenous antioxidant system's quenching capacity, resulting in cumulative oxidative stress. Eventually this causes cell damage which cannot be repaired by internal mechanisms, leading to loss of organ mass and functionality, which ultimately culminates in system dysfunction [3]. Indeed, long-term oxidative stress states have been linked to various diseases associated with advanced age, most notably diabetes, chronic obstructive pulmonary disease (COPD), cardiovascular disease, cancer, diabetes, and asthma [4]. Many of these diseases are not only associated with ageing anymore, but also with the high-obesity, high-stress, and sedentary modern lifestyle (albeit perhaps in milder form) [5]. It is thus vital to address the prevention of these detrimental long-term outcomes, as they are afflicting not only the aged, but also relatively young populations, such as university students [6, 7].

The aetiological mechanism(s) of the various chronic diseases mentioned are each different, as each disease has its own complexities. However, chronic cumulative oxidative stress is a common factor in these diseases, highlighting this system as a vital therapeutic and/or preventative medicine target [8].

Even in the absence of age-associated disease, various theories have linked oxidative stress directly to the normal ageing process. We briefly mention two here. Firstly, the Free Radial Theory of Ageing proposes that the presence of free radicals and their effects on cells are one of the causes of cell ageing and subsequent cell death, which in turn lead to loss of organ mass and other features of whole organism ageing. This theory was first suggested by Harman in 1956 [9], who hypothesized that irradiation of cellular components resulting from the liberation of ${ }^{\circ} \mathrm{OH}$ and ${ }^{\bullet} \mathrm{HO}_{2}$ radicals would lead to dysfunction and mutations which cannot be reversed and thus result in ageing. This theory was later supported and expanded as research began elucidating the details of his proposed theory, for example, by describing the role of SOD [10]. Phenomenally, a very recent metaanalysis [11] confirmed the sustained validity of this theory, 60 years later. Of course, given the technological advances made over this period, it is no surprise that subsequent research has added more detail on mechanisms in support of this theory, although many unresolved questions remain [12].

Secondly, the Replicative Senescence Theory is based on the hypothesis that oxidative stress induces cell death and/or senescence, which necessitates an increase in the rate of cell replication. This in turn accelerates the detrimental effects associated with repeated cycles of mitosis. Telomere length and its accelerated shortening due to reparative replication is the basis of this theory, which was first described by Hayflick in 1965, in response to the observation of a decline in functionality of cell cultures (fibroblasts) which had undergone numerous cell divisions [13]. This phenomenon was subsequently confirmed by experiments on primary peripheral blood mononuclear cells (PBMCs) and fibroblasts from a largely aged population with increased risk for vascular dementia [14]. In this report, decreased telomere length in both fibroblasts and PBMCs was correlated to risk for dementia induced by stroke. In addition, telomere shortening rate was reported to decrease with increasing antioxidant capacity in fibroblasts of the same population. 
This theory, together with the subsequent data, presents evidence that, firstly, oxidative stress is responsible for the accelerated shortening of telomeres brought about by more frequent reparative replication and, secondly, that an increase in antioxidant defence capacity could slow down the ageing process. Note at this point that we do not infer that telomere shortening results only from oxidative stress: other mechanisms such as chromosomal instability have indeed been linked to both ageing and pathological conditions such as cancer [15]. However, to remain focused on the main topic of this review, we have limited this discussion to accelerated telomere shortening in the context of cumulative oxidative stress.

Holistic interpretation of the two theories introduced above implicates oxidative stress as major causative role player in the damage to cell constituents by oxidising membranes, molecules, and DNA. This initiates a cascade of events leading to the need for either cell growth and replication for repair, or death. This implicates ROS as rate determining factor of cell lifespan due to the direct damage it inflicts. Furthermore, oxidative stress is implicated in causing irreversible damage to the mechanism of replication through accelerated telomere shortening and thus ultimately decreases the capacity of the cell to replicate optimally, or at all. With both repair and replication affected, cell senescence is encouraged.

In keeping with the idea of a holistic approach, consideration of oxidative stress in isolation is insufficient. A basic but practical example of the interplay between the oxidative stress system and another system implicated in ageing, inflammation [16], is data available on detrimental effects of cigarette smoking. In this context, both acute smoking and long-term smoking were shown to overwhelm the glutathione antioxidant defence system within the lungs, which was associated with significant infiltration of inflammatory immune cells in the lungs [17]. This study clearly shows system interaction. Furthermore, in the same study, the severity of this maladaptation increased with duration of habitual smoking (in years) and was exacerbated by natural decreases in antioxidant capacity as experienced with ageing, resulting in increased oxidative stress, illustrating the significance of cumulative damage.

However, before considering these interactions in more detail, the literature providing proof of a role for chronic inflammation in the process of ageing will be briefly reviewed.

\section{Chronic Low Grade Inflammation Facilitates Premature Ageing}

As is the case for oxidative stress, the inflammatory response is a system essential for normal body function [18]. As component of the innate immune response, inflammation is a major first-line defence against infection and injury [18]. Apart from this largely independent, nonspecific role in immunity, inflammation is also vital for many specific immune responses to run its course [19]. However, in the process of repair and restoration after insult, the inflammatory response inadvertently disrupts cellular homeostasis of previously unharmed or unaffected cells $[20,21]$. The injuryrepair cycle which inflammation regulates is an efficient system during youth, when optimal sensitivity and response to signalling molecules (such as cytokines, growth factors, prostaglandins, and peptides) maintain the general health of circulating immune cells and the tissue microenvironment, with minimum secondary damage. However, during natural chronological ageing, long-term, repeated stimulusresponse cycles change the receptor expression levels and thus sensitivity to these molecular stimuli [22]. This may necessitate relatively increased concentrations of any particular stimulus to maintain the required effect. Commonly reported characteristics of the natural ageing process include inflammation or oxidative stress-associated symptoms such as directionally inaccurate chemotaxis, premature or suboptimal respiratory burst, and increased proinflammatory signalling from immune cells, all of which form the basis of immunosenescence [23].

Immunosenescence is the term used to describe ageing of immune cells and the functioning of the immune system as a whole. This occurs naturally with advancing chronological age or as result of lifestyle factors, as already mentioned. There is more than one way in which the immune system is compromised upon ageing. Firstly, immunocompetent cells are derived from hematopoietic stem cells. With ageing, a natural bias develops for stem cells to commit to expansion of the myeloid lineage at the cost of the lymphoid lineage [24]. This results in a shift in the balance of immune cells available to enter the circulation. Secondly, the chronic low grade inflammation associated with ageing recruits larger numbers of cells into circulation from the hematopoietic tissue. However, despite the higher circulating cell counts, phagocyte Toll-like receptor expression and phagocytic capacity are decreased in the aged [22], leaving the immune system with a lower capacity for becoming activated and for responding to a more acute insult, such as viral infection. These maladaptations, which together further predispose the individual to proinflammatory responses, are postulated to stem at least in part from alterations in HPA-axis signalling.

The process of immunosenescence may be accelerated by unhealthy lifestyle, such as psychological stress and obesity. As mentioned before, lifestyle-associated diseases share clinical symptoms associated with normal ageing. Indeed rutin, a potent antioxidant, has been shown to protect against ageingrelated metabolic dysfunction [25]. Thus, studies focused on those conditions may provide much insight in terms of ageing and the supplementation options to limit its progress.

For example, in the context of obesity and/or inactivity, cytokines release from adipose tissue macrophages has been demonstrated by many researchers [26-29]. Tumour necrosis factor- $\alpha$ (TNF- $\alpha$ ), interleukin- (IL-) $1 \beta$, and IL6 are among some of the cytokines shown to be released from resident macrophages in adipose tissue, resulting in a proinflammatory microenvironment [30]. Furthermore, 
chronic stress, and in particular psychological stress, is a generally accepted cause of chronic low grade inflammation. For example, in 38 medical students, psychological stress was associated with increased proinflammatory cytokine (TNF$\alpha$, IL-6, IL-1Ra, and IFN- $\gamma$ ) levels, as well as decreased antiinflammatory regulators (IL-10 and IL-4) [31]. Interestingly, these effects were exacerbated by high anxiety proneness as trait, again suggesting that a cumulative stimulus (in this case lifelong anxiety) further exacerbates the undesirable adaptation.

Also in posttraumatic stress disorder (as extreme form of chronic psychological stress), an initial glucocorticoid hyperresponse is followed by glucocorticoid hyporesponsiveness, which is associated by a relatively proinflammatory state. In this condition of continuous proinflammatory signalling, the feedback systems, which usually downregulate inflammation, adjust overtime and result in maladaptations such as chronic but low grade upregulation of proinflammatory mediators (e.g., IL-6, TNF- $\alpha$, IL-1 $\beta$, and prostaglandin $\mathrm{E}_{2}$ ) [32]. Incidentally, the secretion of the first three is mediated by the NF$\kappa \mathrm{B}$ pathway, which is activated in response to cellular stress [33].

In addition to increased proinflammatory signalling, other more mechanical cellular mechanisms also seem to be compromised over time. For example, inappropriate and/or insufficient neutrophil responses result from its decreased phagocytic capacity, increased basal levels of intracellular calcium, and the resulting reduction in capacity for chemotaxis [34]. The result is a chronic low grade inflammatory status in relative absence of a specific threat which can persist for extended periods of time and cause harm and inefficiency of the system. Thus, although varied in specific causative mechanism, the outcome of all of these suboptimal life events or lifestyle habits, for example, chronic stress leading to glucocorticoid resistance and/or cardiovascular disease and high-calorie diets and inactivity (obesity) leading to insulin resistance and diabetes, is that of a chronic inflammatory state [16].

Of particular interest in the context of ageing is the fact that, apart from the now notorious low grade inflammation as primary culprit, this maladaptation results in a compromised capacity to mount an efficient inflammatory response to acute insults. As overviewed in the review by Weinberger and colleagues [35], several reports from clinical literature support the notion that, in the aged individual, that there is a significant increase in the convalescence period required for recovery from injury and pathogen clearance, as well as a decrease in the quality of repair, thus favouring disease progression and morbidity due to injury. Very recently, Baëhl and colleagues (2015) demonstrated, in a longitudinal study of elderly patients, that the acute stress of a hip fracture had a negative effect on neutrophil function immediately after injury. While some neutrophil functions (chemotaxis, phagocytosis) were recovered over time, several others (superoxide production, complement $\mathrm{C} 5 \mathrm{~A}$ and $\mathrm{CD} 11 \mathrm{~b}$ receptor level, and cytokine secretory profile) were still impaired even 6 months after injury. From this, the authors concluded that the acute stress had a long-term negative effect on neutrophil responses, which negatively influenced clinical outcomes, such as the resolution of long-term inflammation, recovery, and susceptibility to opportunistic infections [36].

It is thus clear that ageing is an inflammatory-mediated process. However, from the inflammation/ageing literature, it is clear that inflammation and oxidative stress cannot be separated as causative factors in this context. For example, the ageing-related shift in balance between the glucocorticoid and inflammatory systems has been linked to increased ROS production, which in turn exacerbates low grade immune activation [37].

\section{Links between Inflammation and Oxidative Stress in the Ageing Process: Identifying Therapeutic Targets}

From the above sections it is evident that, in ageing, oxidative stress and inflammation are interdependent mechanisms. We postulate that unravelling and understanding of these intricate links between the two responses hold the answer to identification of the major contributor(s) to allostatic load and maladaptation associated with ageing and age-related pathology.

Generally, repeated exposure to reactive oxygen and nitrogen species causes cell damage and thus a proinflammatory signalling response. For example, in aged mice, unquenched reactive oxygen and nitrogen species act as proinflammatory signalling molecules and mediators of inflammation within the cell itself [38]. More specifically, oxidative damage to cells prompts the release of TNF- $\alpha$ from these damaged cells [21]. Binding of TNF- $\alpha$ to cell surface TNF- $\alpha$ receptors activates the NF- $\kappa$ B inflammasome, which results in the further production of other proinflammatory cytokines, most notably IL- $1 \beta$. Incidentally, TNF$\alpha$ specifically has also been implicated in ROS-mediated upregulation of adhesion molecules which facilitate the infiltration of immune cells into tissue [39], with more on this later. Upregulation of inflammation via the NF- $\kappa \mathrm{B}$ inflammasome is probably the main aetiological mechanism for age-related chronic conditions with an inflammatory component. Indeed, TNF- $\alpha$ upregulation, which is a direct result of increased flux through the inflammasome, has been implicated as causative factor in cardiovascular disease [40]. Of particular relevance to the topic of ageing, the $\mathrm{NF}-\kappa \mathrm{B}$ inflammasome has regulatory roles in cell growth, survival, and proliferation. However, as recently reviewed [41], ROS production may have either inhibitory or stimulatory roles in the NF- $\kappa \mathrm{B}$ pathway, suggesting a dosedependency of the effects of ROS. This unfortunately also means that development of an intervention strategy/product to modulate this target mechanism is no simple feat and will have to be approached in a very tightly controlled "modification range."

From the literature consulted, it is clear that bidirectional communication is in place. For example, both neutrophils and macrophages are producers of oxidants via the NADPHoxidase system [42]. The NADPH-oxidase (NOX) proteins 
aid the transport of electrons across biological membranes and are found in all cells [43]. They are also one of the major generators of ROS in all cells. Particularly, these proteins are the predominant ROS producers in phagocytic cells, a process required for the normal respiratory burst that phagocytes use to kill pathogens and digest cell debris.

Furthermore, activated neutrophils release myeloperoxidase (MPO), which contributes to the formation of hypochlorous acid (HOCL) by acting as a catalyst when reacting with hydrogen peroxide $\left(\mathrm{H}_{2} \mathrm{O}_{2}\right)$. This directly increases the production of ROS [44]. The oxidative burst of neutrophils in itself releases oxidants such as $\mathrm{H}_{2} \mathrm{O}_{2}$, which are harmful to healthy cells and tissue [45]. Besides directly increasing the production of reactive oxygen species itself which causes damage to surrounding cells, MPO specifically has been implicated as a risk factor for coronary artery disease, due to its capacity for oxidation of lipid membranes [46]. The increased oxidant concentration due to immune cell functions, as well as the resultant cell damage, results in increased metabolism in surrounding healthy tissue.

Also at gene level, role players have been elucidated in the context of a ROS-inflammation link. For example, sirtuins are Class III histone deacetylases which are responsible for the deacetylation at $\mathrm{N}$-epsilon lysine residues, a reaction which consumes NAD+. SIRT1 specifically is a sirtuin commonly associated with antioxidant function [47]. Its regulation of oxidative stress is threefold: firstly, it stimulates the expression of antioxidants via the fork-head box protein $\mathrm{O}$ (FOXO) pathway. Secondly, it is involved in inhibiting the NF- $\kappa$ B signalling pathway. In contrast, however, excessive ROS can inhibit SIRT1 activity by oxidatively modifying its cysteine residues and thereby releasing its inhibition of the NF- $\kappa \mathrm{B}$ pathway [47]. It is thus theoretically possible for cumulative stress to downregulate SIRT1 activity in the ageing process. Thirdly, SIRT1 has also been implicated in regulation of apoptosis by deacetylating p53 to inhibit p53dependent transcription in models of cellular stress [48]. This tripartite role defines SIRT1 as another important molecular target in the context of both normal and accelerated ageing.

Apart from these targets related to cellular signalling, cell functional capacity should also be a focus. A striking example of the oxidative stress, inflammation link in ageing, is the decreased capacity for neutrophil chemokinesis reported in the elderly both in terms of motility and accuracy [49]. Normally, immune cells are attracted by chemotactic signals from injured tissue, to migrate to sites of injury or pathogen invasion. During this chemokinetic response, immune cells, typically neutrophils and classically activated macrophages, migrate through tissue toward the site of injury. This movement is facilitated largely via adhesion molecules such as the beta-integrins and I-CAM1 in the case of neutrophils [21]. However, as mentioned earlier, expression of adhesion molecules on neutrophils increases with ageing [39], so that their movement is slowed. In addition, due to yet unclear mechanisms, but most probably due to adaptation of cellular "homing" molecules, directional accuracy of neutrophil migration is also compromised in the elderly. Sapey et al. showed that inaccurate neutrophil migration was causally associated with increased constitutive phosphoinositide 3-kinase (PI3K) signalling [49]. This results not only in inefficient inflammation due to prolonged response time, but the directional inaccuracy of movement also results in mechanical and oxidative damage to relatively more cells in the path of the migrating inflammatory cell [49].

It is clear that both oxidative stress and inflammation are able to induce and exacerbate one another (both indirectly and directly). Furthermore, regardless of the primary signal or which pathway was activated first, these interrelated processes form a vicious cycle which is difficult to target therapeutically because of its complexity. However, it is also this interrelated nature of the two systems that has led us to investigate the possibilities of antioxidants as treatment options to attenuate the cumulative effects of oxidative stress and in turn low grade chronic inflammation.

\section{Are Grapes the Answer to Prevention of Ageing?}

Despite the huge range of nonsteroidal and natural product anti-inflammatories on the market today, the scientific literature shows a conspicuous lack of consistent support for any specific medication. This is perhaps at least in part due to the fact that researches investigating these products cannot keep up with the rate at which new ones are pushed onto the market. Hopefully new legislation on the control of these substances will affect this trend to the benefit of the consumer, by allowing for (or demanding) appropriate testing of these products.

Nevertheless, antioxidants are being used almost routinely by many individuals who wish to supplement for enhancement of general health or as adjuvant therapy in conjunction with more mainstream, pharmaceutical medication. Although they are generally not regarded as a primary therapeutic option, antioxidants may hold particular potential in the realm of preventative medicine. The potential benefits of appropriate antioxidant supplementation are vast, especially when considering the connection between oxidative stress and inflammation. An antioxidant with the capacity to modulate inflammatory status can thus be beneficial to both normal ageing individuals and those suffering from lifestyleassociated diseases.

A comprehensive search of the scientific literature revealed that grape-derived antioxidants are consistently reported to have high benefit and low risk in the context of both oxidative stress and inflammation. These positive results are further strengthened by the fact that these consistent findings were reported across many different models and using a variety of different preparations, ranging from relatively crude extracts to highly purified ones. For example, in terms of purified polyphenols, resveratrol, one of the best-known polyphenols present in grapes as well as 
other plants, is commonly linked to anti-inflammatory [50], antioxidant $[51,52]$, and thus by implication antiageing effects in the scientific literature, as well as anecdotally. Indeed, a recent paper [53] elucidated a role for resveratrol to protect against inflammatory damage via SIRT1 inhibition of the NF$\kappa \mathrm{B}$ pathway (a mechanism discussed above in the context of ageing). In addition, more advanced studies have been undertaken on this polyphenol to better understand the relationship between the chemical structure of resveratrol and its biological activity, especially in terms of oxidant scavenging [54]. Also, pharmaceutical groups have been working on optimisation of delivery systems for resveratrol [55]. Such information may further advance the popularity of this very promising natural product with the pharmaceutical industry, to the benefit of consumers. The phenomenal frequency at which new papers on resveratrol appear, all providing evidence of positive effects in this context, suggests that this particular polyphenol should be investigated in the context of ageing as a matter of urgency.

Even more promising than the many positive effects described for resveratrol above is the fact that resveratrol is only one from a range of equally beneficial substances contained in grapes. The flavonoids quercetin and dihydroquercetin (DHQ), as well as proanthocyanidins and anthocyanins, all of which are present in grapes and a variety of other plant sources, have similarly been linked to both antioxidant and anti-inflammatory effects [50, 52]. To date, despite appearance of a few very promising reports in this context, ageing specifically has not been the focus of many studies investigating these substances. Therefore, for the purpose of this review, we provide a comprehensive overview of the few existing ageing-related studies in this context that were available to us. Results from relevant papers that did not have ageing as a focus were also included, where those results contribute to our understanding of the role of grapederived polyphenols in the oxidative stress, inflammation link in the context of prevention or deceleration of the ageing process.

When considering in vivo studies on ageing as a starting point, resveratrol $(0.1 \mu \mathrm{M}$ to $2.5 \mu \mathrm{M})$ exhibited a clear dosedependent effect on longevity in fish with known short lifespan: resveratrol supplemented fish almost doubled their expected 13-week lifespan and continued to produce healthy offspring long after all control fish had died. Even though resveratrol supplementation was only started in adulthood in this study (i.e., it compares to when humans might start to consider supplementation), it effectively delayed agedependent compromise of locomotor and cognitive performance and reduced expression of neurofibrillary degeneration in the brain [56]. This result of improved neural morphology was recently further substantiated in an aged rat model, where chronic resveratrol treatment prevented detrimental changes in dendritic morphology which is linked not only to ageing, but also to Alzheimer's disease [57]. Similarly, 2 months of ingesting polyphenols in the form of $10 \%$ grape juice resulted in enhanced potassium-evoked dopamine release and cognitive performance in aged rats [58].
A more recent review [59] provides more insight into the potential mechanisms by which age-related cognitive disorders may be curbed by grape polyphenols. Some of these mechanisms at first seem unrelated to the scope of this review, for example, preventing of amyloid-beta deposition associated with Alzheimer's dementia [60]. However, recent research suggests a role for inflammation in the development of the disease [61], while natural antioxidants have been linked to prevention of amyloid-beta deposition [62]. Together, these data suggest that even these seemingly unrelated mechanisms may be interconnected to either inflammation, or oxidative stress, or both. However, more clearly in context of this review, resveratrol was reported to increase NO production, resulting in vasodilation $[59,63]$, which may play a role in the maintenance of central circulation and thus perhaps slower degenerative central processes, as has indeed been reported for resveratrol, as mentioned earlier. However, the role of NO in the context of antioxidant status is much more complex, so that this effect of grape polyphenols should probably receive more attention before it can be interpreted fully in terms of mechanism(s) involved. Furthermore, recently in a coculture simulation of the human blood-brain barrier, another grape polyphenol, proanthocyanidin, was associated with significant inhibition of monocyte infiltration and proinflammatory cytokine secretion in HIV-associated neuroinflammation [64]. Such inhibition of neuroinflammation is associated with a better prognosis in terms of HIV-related neurodegeneration and dementia, further confirming the neuroprotective potential of grapederived antioxidants. Taken together, these studies suggest that the neuroprotective effects of grape polyphenols involve both antioxidant and anti-inflammatory mechanisms, with the latter including not only modulated cytokine signalling, but also modulation of both motility and functional capacity of leukocytes, as previously illustrated by our group $[64,65]$.

One may argue that both these results may be the result of decreased cell activation, perhaps as a result of the known altered cytokine environment. However, an age-associated lack of neutrophil chemokinetic accuracy in response to the chemotaxin fMLP has been reported [49], which suggests that the mechanism is probably related to age-induced compromise of specific cellular mechanisms, rather than activation. In addition, in an ongoing study by our group, we have been able to illustrate by using Dunn chamber chemokinetic assays that grape polyphenols (specifically proanthocyanidin) are able to correct this age-associated anomaly (unpublished data). From Figure 1, which depicts typical digital images obtained for the path of individual neutrophils, it is clear that a more purposeful, directionally accurate movement was achieved in proanthocyanidin-treated neutrophils. This will ensure a more optimal inflammatory response (i.e., the response will be effective, result in relatively insignificant secondary damage, and be resolved in the minimum amount of time). In contrast the rather "aimless wander" of untreated neutrophils from aged individuals will result in not only ineffective immune cell infiltration to sites where they are required, but also relatively more secondary tissue damage and thus prolonged and exacerbated inflammation. 


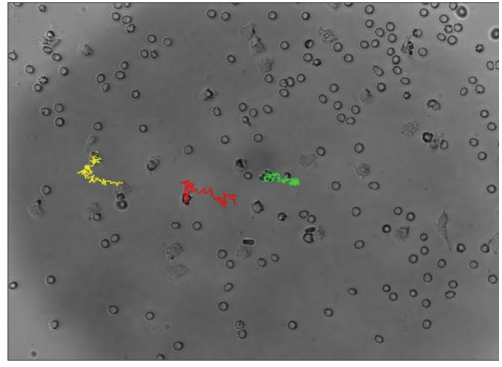

(a)

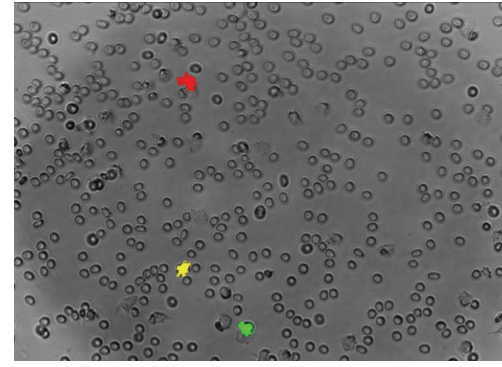

(b)

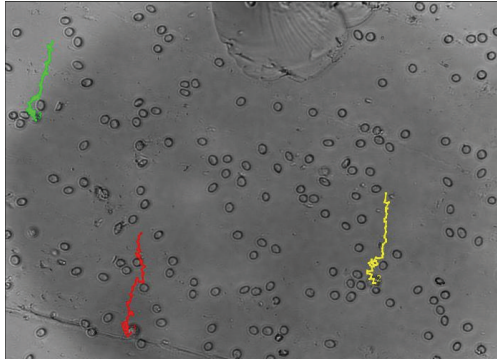

(c)

Figure 1: Typical neutrophil chemotaxis pathways for (a) a young participant ( $<25 \mathrm{yr}$ ), (b) an aged participant ( $>65 \mathrm{yr}$ ), and (c) an aged participant after acute in vitro treatment with grape-deed derived proanthocyanidin. The Olympus Cell ${ }^{\circledR}$ system IX-81 inverted fluorescent microscope system with an F-view cooled CCD camera (Soft Imaging Systems) at 20x magnification was used to capture these images, which was analysed using Image J (Java software).

Turning attention to inflammation and oxidative stress again, the most probable targets for therapeutic intervention in the context of ageing have been described in Section 4. Since the ageing literature is relatively lacking in terms of papers on polyphenol intervention, we have tabulated effects of grape polyphenols reported for these identified targets in Table 1, citing relevant data that was mostly obtained in models other than ageing. The aim with this table was not to present all studies on grape-derived products; rather, it is an attempt to show the many models, species, and disease systems in which beneficial effects on oxidative stress and inflammatory status have consistently been reported. Also, although we have included doses used for in vivo studies for general comparison and again to illustrate the large variations in doses, these doses are product/extract specific and cannot be extrapolated across board.

From Table 1, which is by no means a complete list of all studies reporting on grape-derived substances, grapederived products are undeniably beneficial in limiting the magnitude of the inflammatory response as well as to increase antioxidant activity and seem to have multiple targets.

Of course, when considering potential antiageing modalities, it is also of interest to include evaluation of changes in quality of life. Since ageing is associated with natural muscle wasting or sarcopenia [83], it is important to note that, in this context, supplementation with grape polyphenols $(50 \mathrm{mg} / \mathrm{kg} /$ day) for 4 weeks mitigated skeletal muscle atrophy in a mouse model of chronic inflammation [84]. This was achieved via modulation of two distinct pathways: one directly linked to inflammation (decreased NF- $\kappa \mathrm{B}$ activation) and the other due to antioxidant function (limited ROS-associated mitochondrial damage and caspase- 3 and -9 activation). Since caspase- 3 activation is also a known proapoptotic signal [85], reduced activation and thus apoptosis may result in fewer mitotic cycles. This, in the context of the telomere hypothesis, may point to deceleration of ageing by the polyphenols. Very recently, grape proanthocyanidin treatment in rats was reported to have an antiapoptotic effect which reduced damage after ischemia/reperfusion of the liver [86], which further substantiates this theory.

Interestingly, a study in mice fed a high-fat diet indicated that grape polyphenols may modify gut microbial community structure to result in lower intestinal and systemic inflammation [87]. This extraordinary result serves to remind us of the potential complexity of plant medicines and the requirement for comprehensive investigation of mechanisms of actions and interactions of any potential product via the traditional clinical trial process followed for new pharmaceutical drugs.

\section{Conclusion}

Ageing and accelerated ageing are not new concepts, but rather the norm in modern society. With a population that is growing relatively older due to advances in medicine, we are however pressed for answers on how to alleviate the symptoms or slow the progression of this inevitable phenomenon. From the literature consulted, no negative effects of grape-derived products became evident, while beneficial effects in the context of oxidative stress and inflammation were consistently reported in the context of numerous cellular targets. Huge variation in product content and prescribed dosage complicates interpretation of the fast growing body of literature on this topic. A recommendation for future studies is the inclusion of more parameters per study, so that a more comprehensive interpretation of specific mechanisms becomes possible. Measurement of only basic indicators of either antioxidant status or inflammatory status, while providing proof of efficacy of the product, does not contribute much information on its mechanism of action. Despite this shortcoming, the literature clearly indicates that grape-derived products are undeniably a therapeutic force to be reckoned in the combat of ageing and age-associated conditions.

\section{Conflict of Interests}

The authors declare that there is no conflict of interests regarding the publication of this paper. 


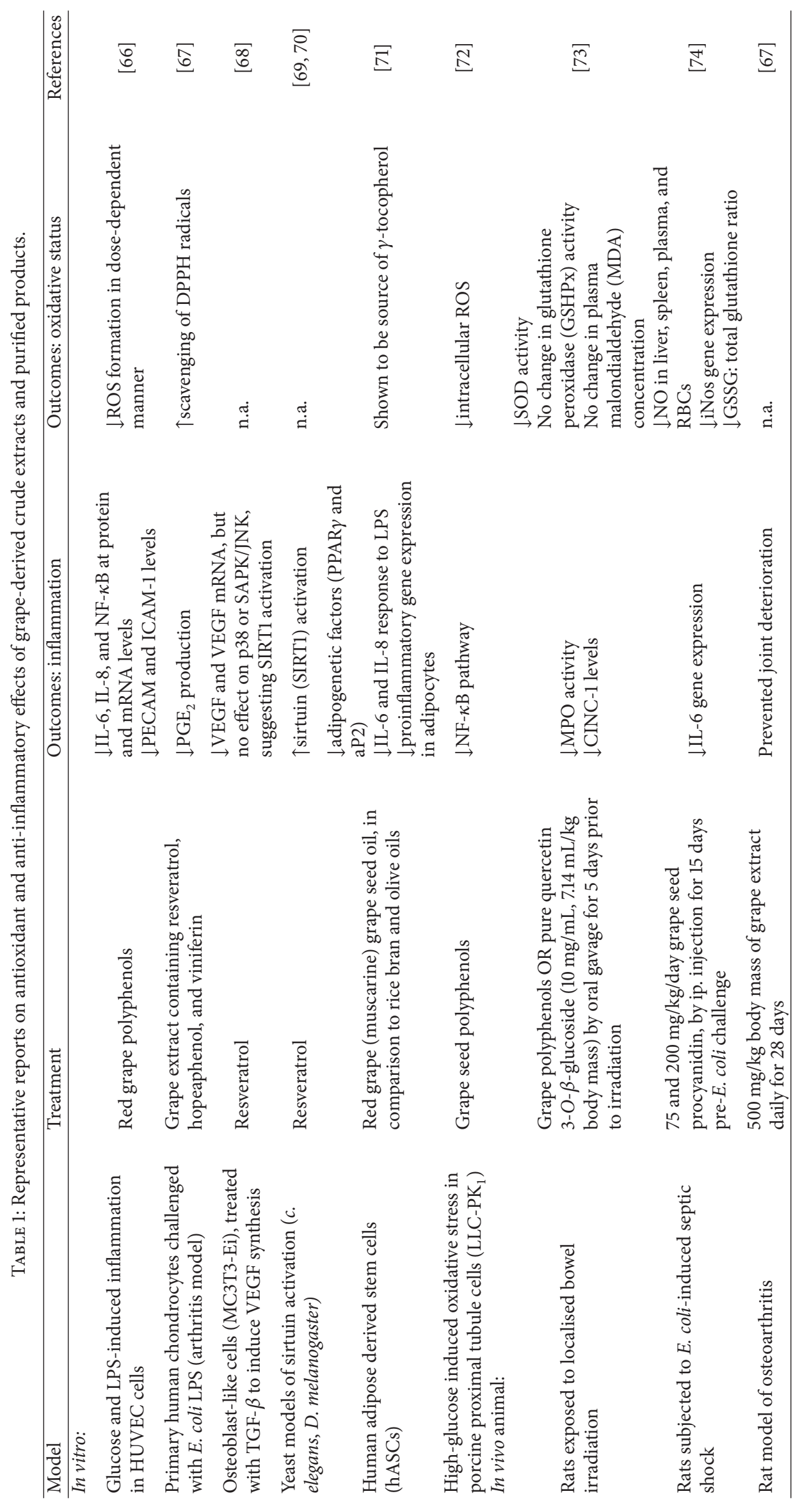




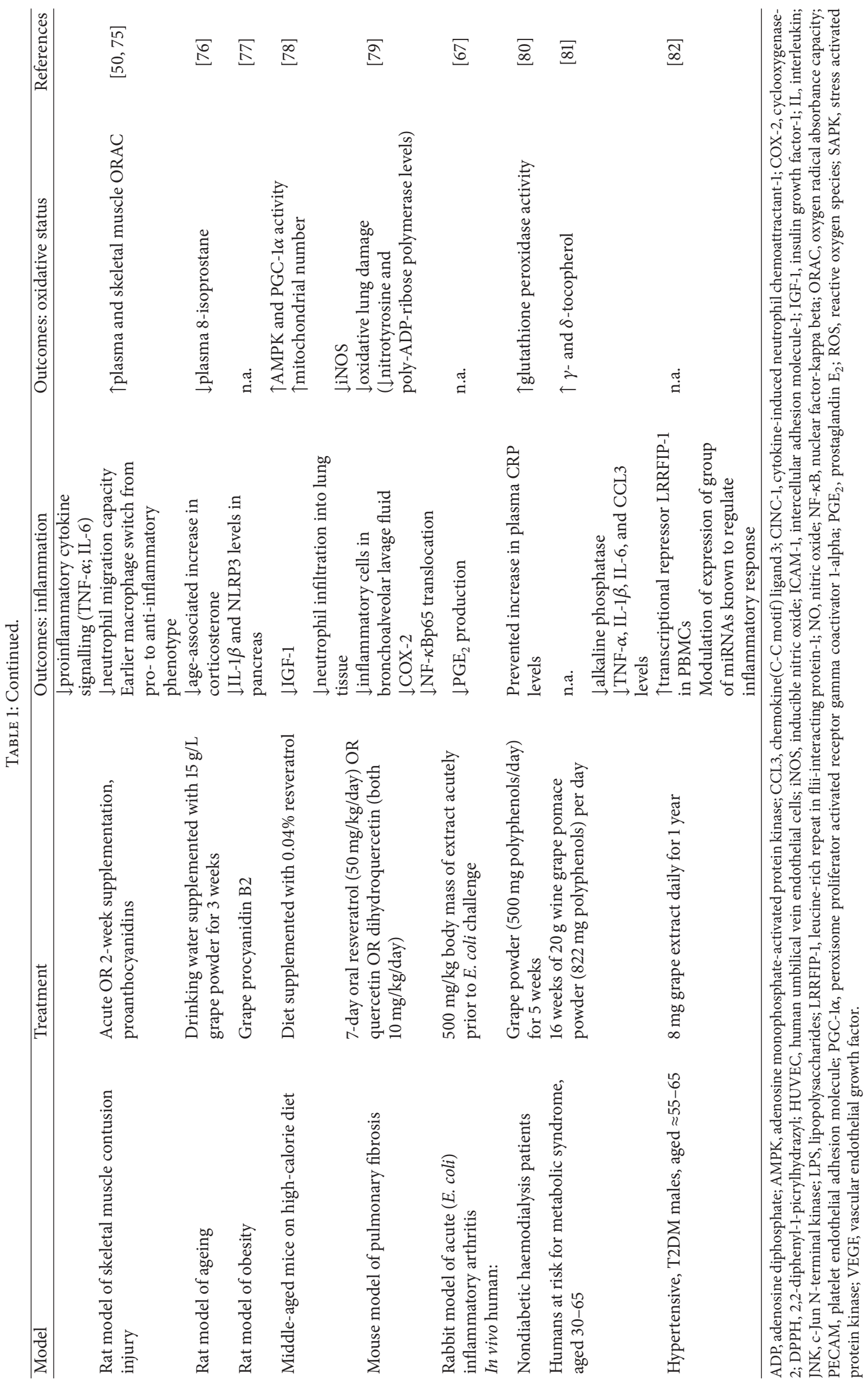




\section{References}

[1] WHO, WHO Fact Sheet, WHO, 2015, http://www.who.int.

[2] B. F. Oliveira, J. A. Nogueira-Machado, and M. M. Chaves, "The role of oxidative stress in the aging process," TheScientificWorldJOURNAL, vol. 10, pp. 1121-1128, 2010.

[3] D. P. Jones, V. C. Mody Jr., J. L. Carlson, M. J. Lynn, and P. Sternberg Jr., "Redox analysis of human plasma allows separation of pro-oxidant events of aging from decline in antioxidant defenses," Free Radical Biology \& Medicine, vol. 33, no. 9, pp. 1290-1300, 2002.

[4] W. MacNee, R. A. Rabinovich, and G. Choudhury, "Ageing and the border between health and disease," The European Respiratory Journal, vol. 44, no. 5, pp. 1332-1352, 2014.

[5] E. Ekblom-Bak, B. Ekblom, M. Vikström, U. De Faire, and M.L. Hellénius, "The importance of non-exercise physical activity for cardiovascular health and longevity," British Journal of Sports Medicine, vol. 48, no. 3, pp. 233-238, 2014.

[6] C. Smith and M. F. Essop, "Gender differences in metabolic risk factor prevalence in a South African student population," Cardiovascular Journal of Africa, vol. 20, no. 3, pp. 178-182, 2009.

[7] C. E. Ottobelli, W. M. De Souza, T. Paz da Silva, R. N. Moresco, and M. B. Moretto, "Adipocytokines, inflammatory and oxidative stress markers of clinical relevance altered in young overweight/obese subjects," Clinical Biochemistry, 2016.

[8] K. Venkataraman, S. Khurana, and T. C. Tai, "Oxidative stress in aging-matters of the heart and mind," International Journal of Molecular Sciences, vol. 14, no. 9, pp. 17897-17925, 2013.

[9] D. Harman, "Aging: a theory based on free radical and radiation chemistry," Journal of gerontology, vol. 11, no. 3, pp. 298-300, 1956.

[10] J. M. McCord and I. Fridovich, "Superoxide dismutase. An enzymic function for erythrocuprein (hemocuprein)," The Journal of Biological Chemistry, vol. 244, no. 22, pp. 6049-6055, 1969.

[11] B. Schöttker, H. Brenner, E. Jansen et al., "Evidence for the free radical/oxidative stress theory of ageing from the CHANCES consortium: a meta-analysis of individual participant data," BMC Medicine, vol. 13, no. 300, 15 pages, 2015.

[12] B. A. I. Payne and P. F. Chinnery, "Mitochondrial dysfunction in aging: much progress but many unresolved questions," Biochimica et Biophysica Acta, vol. 1847, no. 11, pp. 1347-1353, 2015.

[13] L. Hayflick, "The limited in vitro lifetime of human diploid cell strains," Experimental Cell Research, vol. 37, no. 3, pp. 614-636, 1965.

[14] T. von Zglinicki, V. Serra, M. Lorenz et al., "Short telomeres in patients with vascular dementia: an indicator of low antioxidative capacity and a possible risk factor?" Laboratory Investigation, vol. 80, no. 11, pp. 1739-1747, 2000.

[15] S. Venkatesan, A. T. Natarajan, and M. P. Hande, "Chromosomal instability-mechanisms and consequences," Mutation Research/Genetic Toxicology and Environmental Mutagenesis, vol. 793, pp. 176-184, 2015.

[16] N. Khansari, Y. Shakiba, and M. Mahmoudi, "Chronic inflammation and oxidative stress as a major cause of age-related diseases and cancer," Recent Patents on Inflammation \& Allergy Drug Discovery, vol. 3, no. 1, pp. 73-80, 2009.

[17] K. Nagai, T. Betsuyaku, T. Kondo, Y. Nasuhara, and M. Nishimura, "Long term smoking with age builds up excessive oxidative stress in bronchoalveolar lavage fluid," Thorax, vol. 61, no. 6, pp. 496-502, 2006.
[18] L. Sherwood, Human Physiology: From Cells to Systems, Brooks/Cole, 7th edition, 2010.

[19] M. E. Kotas and R. Medzhitov, "Homeostasis, inflammation, and disease susceptibility," Cell, vol. 160, no. 5, pp. 816-827, 2015.

[20] J. G. Tidball, "Inflammatory processes in muscle injury and repair," American Journal of Physiology-Regulatory Integrative and Comparative Physiology, vol. 288, no. 2, pp. R345-R353, 2005.

[21] C. Smith, M. J. Kruger, R. M. Smith, and K. H. Myburgh, “The inflammatory response to skeletal muscle injury: illuminating complexities," Sports Medicine, vol. 38, no. 11, pp. 947-969, 2008.

[22] A. Panda, A. Arjona, E. Sapey et al., "Human innate immunosenescence: causes and consequences for immunity in old age," Trends in Immunology, vol. 30, no. 7, pp. 325-333, 2009.

[23] T. Fulop, A. Larbi, N. Douziech et al., "Signal transduction and functional changes in neutrophils with aging," Aging Cell, vol. 3, no. 4, pp. 217-226, 2004.

[24] I. Beerman, D. Bhattacharya, S. Zandi et al., "Functionally distinct hematopoietic stem cells modulate hematopoietic lineage potential during aging by a mechanism of clonal expansion," Proceedings of the National Academy of Sciences of the United States of America, vol. 107, no. 12, pp. 5465-5470, 2010.

[25] T. Li, S. Chen, T. Feng, J. Dong, Y. Li, and H. Li, "Rutin protects against aging-related metabolic dysfunction," Food \& Function, 2016.

[26] A. A. Hill, E. K. Anderson-Baucum, A. J. Kennedy et al., "Activation of NF- $\kappa \mathrm{B}$ drives the enhanced survival of adipose tissue macrophages in an obesogenic environment," Molecular Metabolism, vol. 4, no. 10, pp. 665-677, 2015.

[27] J. Y. Huh, Y. J. Park, M. Ham, and J. B. Kim, "Crosstalk between adipocytes and immune cells in adipose tissue inflammation and metabolic dysregulation in obesity," Molecules and Cells, vol. 37, no. 5, pp. 365-371, 2014.

[28] S. P. Weisberg, D. McCann, M. Desai, M. Rosenbaum, R. L. Leibel, and A. W. Ferrante Jr., "Obesity is associated with macrophage accumulation in adipose tissue," The Journal of Clinical Investigation, vol. 112, no. 12, pp. 1796-1808, 2003.

[29] D. C. K. Borato, G. C. Parabocz, J. T. Ribas et al., "Biomarkers in obesity: serum myeloperoxidase and traditional cardiac risk parameters," Experimental and Clinical Endocrinology \& Diabetes, vol. 124, no. 1, pp. 49-54, 2016.

[30] H. Khodabandehloo, S. Gorgani-Firuzjaee, G. Panahi, and R. Meshkani, "Molecular and cellular mechanisms linking inflammation to insulin resistance and $\beta$-cell dysfunction," Translational Research, vol. 167, no. 1, pp. 228-256, 2016.

[31] M. Maes, C. Song, A. Lin et al., "The effects of psychological stress on humans: increased production of pro-inflammatory cytokines and a Th1-like response in stress-induced anxiety," Cytokine, vol. 10, no. 4, pp. 313-318, 1998.

[32] H. Bruunsgaard, "The clinical impact of systemic low-level inflammation in elderly populations," Danish Medical Bulletin, vol. 53, no. 3, pp. 285-309, 2006.

[33] N. Sallam and I. Laher, "Exercise modulates oxidative stress and inflammation in aging and cardiovascular diseases," Oxidative Medicine and Cellular Longevity, vol. 2016, Article ID 7239639, 32 pages, 2016.

[34] C. Wenisch, S. Patruta, F. Daxböck, R. Krause, and W. Hörl, "Effect of age on human neutrophil function," Journal of Leukocyte Biology, vol. 67, no. 1, pp. 40-45, 2000.

[35] B. Weinberger, D. Herndler-Brandstetter, A. Schwanninger, D. Weiskopf, and B. Grubeck-Loebenstein, "Biology of immune 
responses to vaccines in elderly persons," Clinical Infectious Diseases, vol. 46, no. 7, pp. 1078-1084, 2008.

[36] S. Baëhl, H. Garneau, A. Le Page et al., "Altered neutrophil functions in elderly patients during a 6-month follow-up period after a hip fracture," Experimental Gerontology, vol. 65, pp. 5868, 2015.

[37] P. H. Naccache and J. S. Lefebvre, "A straight neutrophil path to healthy aging?” Blood, vol. 123, no. 2, pp. 154-156, 2014.

[38] M. Mittal, M. R. Siddiqui, K. Tran, S. P. Reddy, and A. B. Malik, "Reactive oxygen species in inflammation and tissue injury," Antioxidants \& Redox Signaling, vol. 20, no. 7, pp. 1126-1167, 2014.

[39] H. Kim, J.-S. Hwang, C.-H. Woo et al., “TNF- $\alpha$-induced upregulation of intercellular adhesion molecule- 1 is regulated by a Rac-ROS-dependent cascade in human airway epithelial cells," Experimental and Molecular Medicine, vol. 40, no. 2, pp. 167-175, 2008.

[40] H. Zhang, Y. Park, J. Wu et al., "Role of TNF- $\alpha$ in vascular dysfunction," Clinical Science, vol. 116, no. 3, pp. 219-230, 2009.

[41] M. J. Morgan and Z.-G. Liu, "Crosstalk of reactive oxygen species and NF- $\kappa \mathrm{B}$ signaling," Cell Research, vol. 21, no. 1, pp. 103-115, 2011.

[42] T. Finkel and N. J. Holbrook, "Oxidants, oxidative stress and the biology of ageing," Nature, vol. 408, no. 6809, pp. 239-247, 2000.

[43] K. Bedard and K.-H. Krause, "The NOX family of ROSgenerating NADPH oxidases: physiology and pathophysiology," Physiological Reviews, vol. 87, no. 1, pp. 245-313, 2007.

[44] A. P. Levine, M. R. Duchen, S. de Villiers, P. R. Rich, A. W. Segal, and A. Gaggar, "Alkalinity of neutrophil phagocytic vacuoles is modulated by $\mathrm{HVCN} 1$ and has consequences for myeloperoxidase activity," PLoS ONE, vol. 10, no. 4, Article ID e0125906, pp. 1-20, 2015.

[45] M. J. Berridge, "Module 11: cell stress, inflammatory responses and cell death," Cell Signalling Biology, 2014.

[46] R. Zhang, M.-L. Brennan, X. Fu et al., "Association between myeloperoxidase levels and risk of coronary artery disease," The Journal of the American Medical Association, vol. 286, no. 17, pp. 2136-2142, 2001.

[47] A. Salminen, K. Kaarniranta, and A. Kauppinen, "Crosstalk between oxidative stress and SIRT1: impact on the aging process," International Journal of Molecular Sciences, vol. 14, no. 2, pp. 3834-3859, 2013.

[48] N. Poulose and R. Raju, "Sirtuin regulation in aging and injury," Biochimica et Biophysica Acta (BBA)-Molecular Basis of Disease, vol. 1852, no. 11, pp. 2442-2455, 2015.

[49] E. Sapey, H. Greenwood, G. Walton et al., "Phosphoinositide 3-kinase inhibition restores neutrophil accuracy in the elderly: toward targeted treatments for immunosenescence," Blood, vol. 123, no. 2, pp. 239-248, 2014.

[50] M. J. Kruger and C. Smith, "Postcontusion polyphenol treatment alters inflammation and muscle regeneration," Medicine and Science in Sports and Exercise, vol. 44, no. 5, pp. 872-880, 2012.

[51] L. Carrera-Quintanar, L. Funes, N. Vicente-Salar et al., "Effect of polyphenol supplements on redox status of blood cells: a randomized controlled exercise training trial," European Journal of Nutrition, vol. 54, no. 7, pp. 1081-1093, 2015.

[52] M. R. De Oliveira, S. F. Nabavi, A. Manayi, M. Daglia, Z. Hajheydari, and S. M. Nabavi, "Resveratrol and the mitochondria: from triggering the intrinsic apoptotic pathway to inducing mitochondrial biogenesis, a mechanistic view," Biochimica et
Biophysica Acta-General Subjects, vol. 1860, no. 4, pp. 727-745, 2016.

[53] W. Pan, H. Yu, S. Huang, P. Zhu, and A. Kumar, "Resveratrol protects against TNF- $\alpha$-induced injury in human umbilical endothelial cells through promoting sirtuin-1-induced repression of NF-KB and p38 MAPK," PLOS ONE, vol. 11, no. 1, Article ID e0147034, 2016.

[54] P. Kotora, F. Šeršeň, J. Filo, D. Loos, J. Gregáň, and F. Gregáň, "The scavenging of DPPH, galvinoxyl and ABTS radicals by imine analogs of resveratrol," Molecules, vol. 21, no. 2, 2016.

[55] G. F. Balata, E. A. Essa, H. A. Shamardl, S. Zidan, and M. Abdo Rehab, "Self-emulsifying drug delivery systems as a tool to improve solubility and bioavailability of resveratrol," Drug Design, Development and Therapy, vol. 10, pp. 117-128, 2016.

[56] D. R. Valenzano, E. Terzibasi, T. Genade, A. Cattaneo, L. Domenici, and A. Cellerino, "Resveratrol prolongs lifespan and retards the onset of age-related markers in a short-lived vertebrate," Current Biology, vol. 16, no. 3, pp. 296-300, 2006.

[57] E. M. Hernández-Hernández, C. Serrano-García, R. A. Vázquez-Roque et al., "Chronic administration of resveratrol prevents morphological changes in prefrontal cortex and hippocampus of aged rats," Synapse, 2016.

[58] B. Shukitt-Hale, A. Carey, L. Simon, D. A. Mark, and J. A. Joseph, "Effects of Concord grape juice on cognitive and motor deficits in aging," Nutrition, vol. 22, no. 3, pp. 295-302, 2006.

[59] E. P. Cherniack, "A berry thought-provoking idea: the potential role of plant polyphenols in the treatment of age-related cognitive disorders," The British Journal of Nutrition, vol. 108, no. 5, pp. 794-800, 2012.

[60] Y.-J. Wang, P. Thomas, J.-H. Zhong et al., "Consumption of grape seed extract prevents amyloid- $\beta$ deposition and attenuates inflammation in brain of an Alzheimer's disease mouse," Neurotoxicity Research, vol. 15, no. 1, pp. 3-14, 2009.

[61] M. C. Kizilarslanoglu, Ö. Kara, Y. Yesil et al., "Alzheimer disease, inflammation, and novel inflammatory marker: resistin," Turkish Journal of Medical Sciences, vol. 45, pp. 1040-1046, 2015.

[62] M. Awasthi, S. Singh, V. P. Pandey, and U. N. Dwivedi, "Alzheimer's disease: an overview of amyloid beta dependent pathogenesis and its therapeutic implications along with in silico approaches emphasizing the role of natural products," Journal of the Neurological Sciences, vol. 361, pp. 256-271, 2016.

[63] F. Cacciapuoti, "Opposite effects of metabolic syndrome and calorie restriction on thrombotic disease: heads and tails of the same coin-resveratrol's role," Metabolic Syndrome and Related Disorders, vol. 7, no. 5, pp. 397-400, 2009.

[64] L. D. Africa and C. Smith, "Using a simulated blood-brain barrier to investigate potential modulators of HIV-1-associated neuro-inflammatory processes in vitro," Journal of Research in Biology, vol. 5, pp. 5-20, 2015.

[65] M. J. Kruger, K. H. Myburgh, and C. Smith, "Contusion injury with chronic in vivo polyphenol supplementation: leukocyte responses," Medicine \& Science in Sports and Exercise, vol. 46, no. 2, pp. 225-231, 2014.

[66] G. D. Noratto, G. Angel-Morales, S. T. Talcott, and S. U. Mertens-Talcott, "Polyphenolics from Açaí (Euterpe oleracea Mart.) and red muscadine grape (Vitis rotundifolia) protect human umbilical vascular endothelial cells (HUVEC) from glucose- and lipopolysaccharide (LPS)-induced inflammation and target microRNA-126," Journal of Agricultural and Food Chemistry, vol. 59, no. 14, pp. 7999-8012, 2011.

[67] C.-F. Tsai, K.-T. Wang, L.-G. Chen, C.-J. Lee, S.-H. Tseng, and C.-C. Wang, "Anti-inflammatory effects of Vitis thunbergii var. 
taiwaniana on knee damage associated with arthritis," Journal of Medicinal Food, vol. 17, no. 4, pp. 479-486, 2014.

[68] G. Kuroyanagi, T. Otsuka, N. Yamamoto, R. MatsushimaNishiwaki, O. Kozawa, and H. Tokuda, "Resveratrol suppresses TGF- $\beta$-induced VEGF synthesis in osteoblasts: inhibition of the p44/p42 MAPKs and SAPK/JNK pathways," Experimental and Therapeutic Medicine, vol. 9, no. 6, pp. 2303-2310, 2015.

[69] J. G. Wood, B. Rogina, S. Lavu et al., "Sirtuin activators mimic caloric restriction and delay ageing in metazoans," Nature, vol. 430, no. 7000, pp. 686-689, 2004.

[70] K. T. Howitz, K. J. Bitterman, H. Y. Cohen et al., "Small molecule activators of sirtuins extend Saccharomyces cerevisiae lifespan," Nature, vol. 425, no. 6954, pp. 191-196, 2003.

[71] L. Zhao, Y. Yagiz, C. Xu, J. Lu, S. Chung, and M. R. Marshall, "Muscadine grape seed oil as a novel source of tocotrienols to reduce adipogenesis and adipocyte inflammation," Food \& Function, vol. 6, no. 7, pp. 2293-2302, 2015.

[72] H. Fujii, T. Yokozawa, Y. A. Kim, C. Tohda, and G.-I. Nonaka, "Protective effect of grape seed polyphenols against high glucose-induced oxidative stress," Bioscience, Biotechnology, and Biochemistry, vol. 70, no. 9, pp. 2104-2111, 2006.

[73] L. Younes-Sakr, P. Senesse, C. Laurent et al., "Validation of a surgical technique for rat intestinal irradiation: potential Side effects prevention by dietary grape phenolics," Digestive Diseases and Sciences, vol. 57, no. 10, pp. 2562-2570, 2012.

[74] V. Pallarès, A. Fernández-Iglesias, L. Cedó et al., "Grape seed procyanidin extract reduces the endotoxic effects induced by lipopolysaccharide in rats," Free Radical Biology \& Medicine, vol. 60, pp. 107-114, 2013.

[75] K. H. Myburgh, M. J. Kruger, and C. Smith, "Accelerated skeletal muscle recovery after in vivo polyphenol administration," Journal of Nutritional Biochemistry, vol. 23, no. 9, pp. 1072-1079, 2012.

[76] G. Patki, Q. Ali, I. Pokkunuri et al., "Grape powder treatment prevents anxiety-like behavior in a rat model of aging," Nutrition Research, vol. 35, no. 6, pp. 504-511, 2015.

[77] W. Yin, B. Li, X. Li et al., "Anti-inflammatory effects of grape seed procyanidin B2 on a diabetic pancreas," Food \& Function, vol. 6, no. 9, pp. 3065-3071, 2015.

[78] J. A. Baur, K. J. Pearson, N. L. Price et al., "Resveratrol improves health and survival of mice on a high-calorie diet," Nature, vol. 444, no. 7117, pp. 337-342, 2006.

[79] D. Impellizzeri, E. Talero, R. Siracusa et al., "Protective effect of polyphenols in an inflammatory process associated with experimental pulmonary fibrosis in mice," The British Journal of Nutrition, vol. 114, no. 6, pp. 853-865, 2015.

[80] A. G. Janiques, V. d. Leal, M. B. Stockler-Pinto, N. X. Moreira, and D. Mafra, "Effects of grape powder supplementation on inflammatory and antioxidant markers in hemodialysis patients: a randomized double-blind study," Jornal Brasileiro de Nefrologia, vol. 36, no. 4, pp. 496-501, 2014.

[81] I. Urquiaga, S. D’Acuña, D. Pérez et al., "Wine grape pomace flour improves blood pressure, fasting glucose and protein damage in humans: a randomized controlled trial," Biological Research, vol. 48, article 49, 2015.

[82] J. Tomé-Carneiro, M. Larrosa, M. J. Yáñez-Gascón et al., "One-year supplementation with a grape extract containing resveratrol modulates inflammatory-related microRNAs and cytokines expression in peripheral blood mononuclear cells of type 2 diabetes and hypertensive patients with coronary artery disease," Pharmacological Research, vol. 72, pp. 69-82, 2013.
[83] M. Zamboni, G. Mazzali, F. Fantin, A. Rossi, and V. Di Francesco, "Sarcopenic obesity: a new category of obesity in the elderly," Nutrition, Metabolism \& Cardiovascular Diseases, vol. 18, no. 5, pp. 388-395, 2008.

[84] K. Lambert, M. Coisy-Quivy, C. Bisbal et al., "Grape polyphenols supplementation reduces muscle atrophy in a mouse model of chronic inflammation," Nutrition, vol. 31, no. 10, pp. 12751283, 2015.

[85] S. W. Lee, G. Dai, Z. Hu, X. Wang, J. Du, and W. E. Mitch, "Regulation of muscle protein degradation: coordinated control of apoptotic and ubiquitin-proteasome systems by phosphatidylinositol 3 kinase," Journal of the American Society of Nephrology, vol. 15, no. 6, pp. 1537-1545, 2004.

[86] Z. C. Xu, J. Yin, B. Zhou et al., "Grape seed proanthocyanidin protects liver against ischemia/reperfusion injury by attenuating endoplasmic reticulum stress," World Journal of Gastroenterology, vol. 21, no. 24, pp. 7468-7477, 2015.

[87] D. E. Roopchand, R. N. Carmody, P. Kuhn et al., "Dietary polyphenols promote growth of the gut bacterium Akkermansia muciniphila and attenuate high-fat diet-induced metabolic syndrome," Diabetes, vol. 64, no. 8, pp. 2847-2858, 2015. 


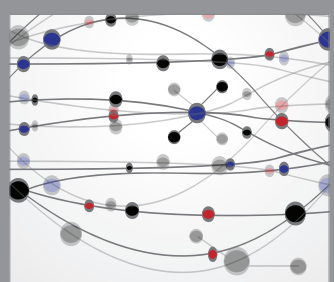

The Scientific World Journal
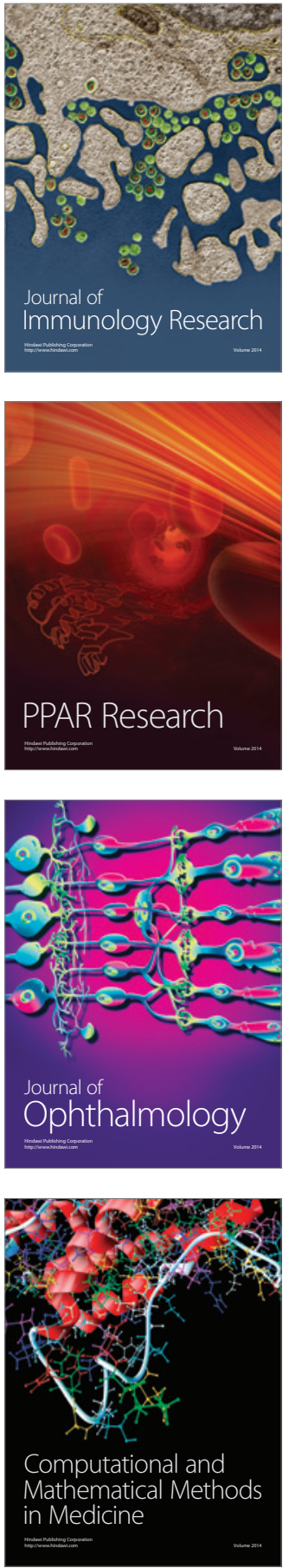

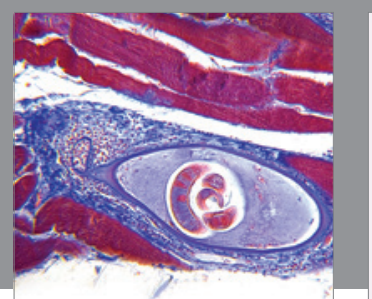

Gastroenterology Research and Practice

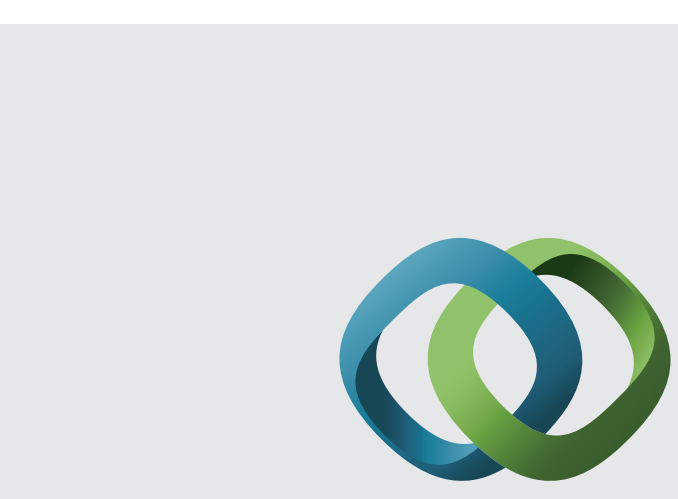

\section{Hindawi}

Submit your manuscripts at

http://www.hindawi.com
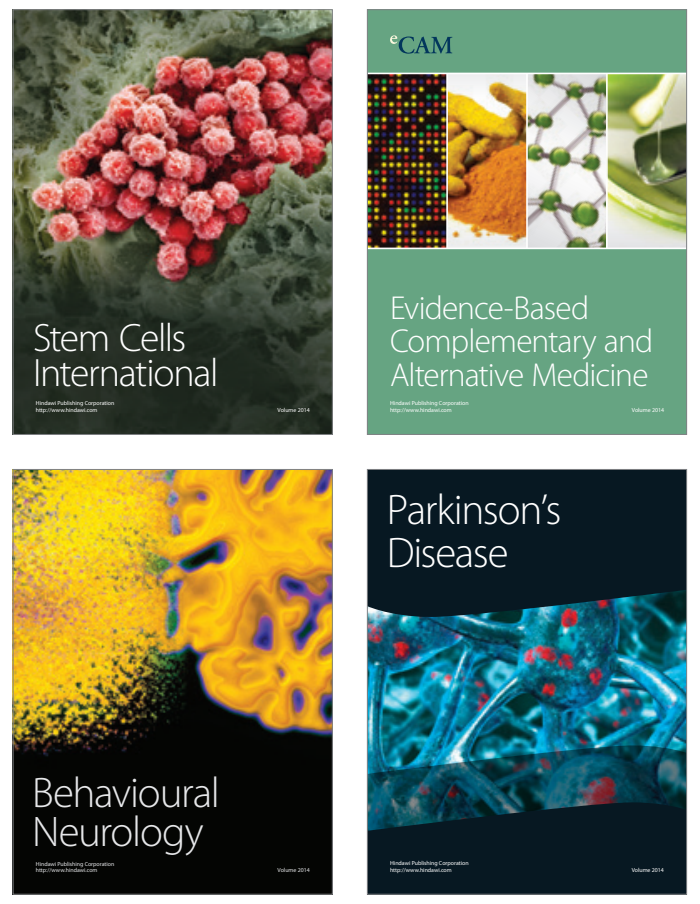
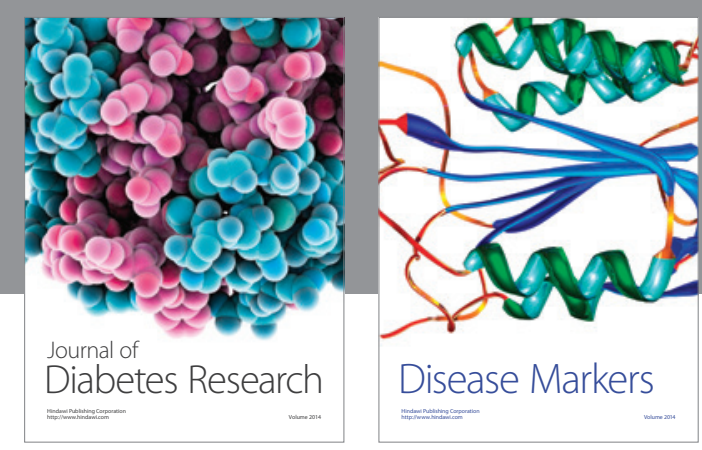

Disease Markers
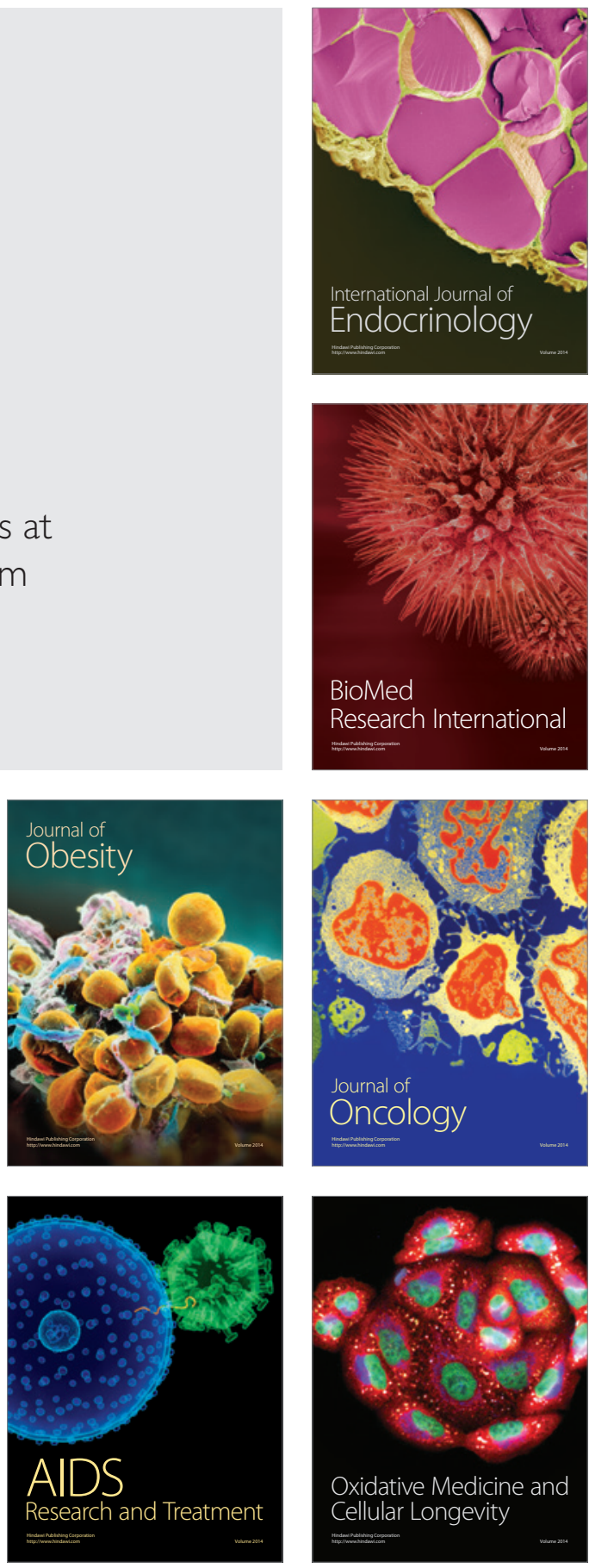